\title{
EXPLORACIÓN DE PATRONES DE INTERACCIÓN PARA SU USO EN LA WEB SEMANTICA
}

\author{
Exploring interaction patterns for use on the \\ semantic web
}

Alfons Palacios, Roberto García, Marta Oliva y Toni Granollers

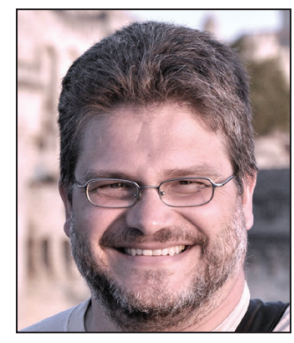

Alfons Palacios es profesor titular de la Escola Superior Politècnica del Tecnocampus Mataró, centro adscrito a la Universitat Pompeu Fabra. Es miembro investigador de web semántica e interacción persona ordenador en Griho (Grupo de investigación en interacción persona ordenador e integración de datos) de la Universitat de Lleida. Licenciado en informática y diplomado en estudios avanzados en software por la Universitat Politècnica de Catalunya, es estudiante de doctorado en la Universitat de Lleida. Es miembro de AIPO (Asociación Interacción Persona Ordenador). http://orcid.org/0000-0003-3546-895X

Universitat Pompeu Fabra, Escola Superior Politècnica. TecnoCampus Mataró-Maresme Av. Ernest Lluch, 32 (Porta Laietana). 08302 Mataró (Barcelona), España palacios@tecnocampus.cat

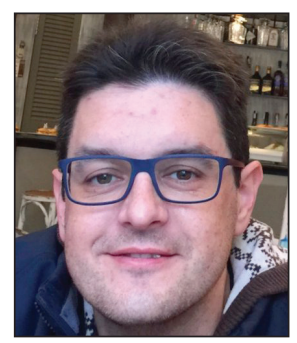

Roberto García es profesor asociado de la Universitat de Lleida (UdL) y director del Griho (Grupo de investigación en interacción persona ordenador e integración de datos). Es autor de más de 50 publicaciones en revistas académicas, actas de congresos y libros, originando más de mil citas en Google Scholar. Su investigación se extiende de la gestión de medios de comunicación a la interacción persona-ordenador, pero siempre intentando aplicar tecnologías semánticas y contribuyendo al despliegue de la web de linked data.

http://rhizomik.net/ roberto

http://orcid.org/0000-0003-2207-9605

Universitat de Lleida Jaume II, 69. 25001 Lleida, España rgarcia@diei.udl.cat

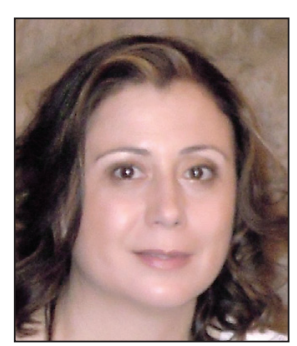

Marta Oliva es profesora asociada de la Universitat de Lleida (UdL) y miembro de Griho (Grupo de investigación en interacción persona ordenador e integración de datos). Obtuvo su MSc. en computer science en la Universitat Politècnica de Catalunya (UPC) donde llevó a cabo su investigación en el proyecto Bloom (Barcelona object oriented model) y completó su tesis sobre Integrating security criteria to achieve access control in a federated heterogeneous database system. En 2005 comenzó su actividad de investigación en los campos de la interacción persona-ordenador y de la web semántica.

http://griho.udl.cat/about/members/oliva-marta.html

http://orcid.org/0000-0002-9329-7282

Universitat de Lleida Jaume II 69, E-25001 Lleida, España oliva@diei.udl.cat

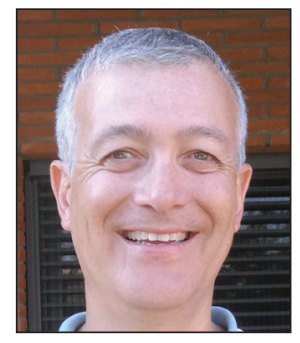

Toni Granollers es profesor asociado de la Universitat de Lleida (UdL) donde realiza actividades docentes y de investigación relacionadas con la interacción persona-ordenador (IPO). Es miembro del Griho (Grupo de investigación en interacción persona ordenador e integración de datos). Obtuvo su MSc en computer science en la Universitat Autònoma de Barcelona (UAB) y su PhD en computer science, especializado en IPO, por la UdL. Es miembro de AIPO (Asociación Interacción Persona-Ordenador), del capítulo $\mathrm{HCl}$ de ACM (ACM-Sigchi) y de IEEE.

http://griho.udl.cat/about/members/granollers-toni.html

http://orcid.org/0000-0001-9189-7308

Universitat de Lleida Jaume II, 69. 25001 Lleida, España tonig@diei.udl.cat 


\title{
Resumen
}

En los últimos años se han publicado múltiples propuestas de conjuntos de patrones de interacción aplicables al diseño y al desarrollo de interfaces web. Paralelamente, la web semántica (WS) mejora los sitios web con contenidos semánticos. Este cambio de paradigma permite cuestionarse las tareas de usuario final, que pueden, para la WS, permanecer invariables, cambiar por completo; o plantear nuevas tareas. A partir de estudios preliminares, se ha definido una lista de tareas elementales de usuario final para la WS. Después, se ha construido un inventario de las principales propuestas de patrones de interacción, que se han clasificado de acuerdo con las tareas de usuario final (indicando dónde pueden ser utilizados). Finalmente se ha analizado cómo los patrones de interacción pueden mejorar la funcionalidad de cada una de las nuevas tareas de usuario final en el contexto de la WS.

\section{Palabras clave}

Web semántica; Interacción persona ordenador; Patrones de interacción; Interfaces de usuario; Gestión y generación de interfaces de usuario; Usuario final; Usabilidad.

\begin{abstract}
In recent years, multiple sets of interaction patterns have been proposed for use in the design and the development of website interfaces. The semantic web also has enhanced websites with semantic content. This paradigm shift allows the reexamination of usual end-user tasks, which, as semantic web end-user tasks, might remain the same, be completely changed, or give rise to the development of new tasks. We have defined a list of semantic web end-user tasks and built an inventory of most of the interaction patterns proposed for semantic web use. We then classified these patterns according to the semantic web end-user tasks where they can be used and analyzed how interaction patterns can improve the functionality of each of those semantic web end-user tasks.
\end{abstract}

\section{Keywords}

Human computer interaction; $\mathrm{HCl}$; Semantic web; Interaction patterns; End-user tasks; Semantic web usability; Graphical user interfaces; GUI.

Palacios, Alfons; García, Roberto; Oliva, Marta; Granollers, Toni (2015). “Exploración de patrones de interacción para su uso en la web semántica". El profesional de la información, v. 24, n. 6, pp. 749-758.

http://dx.doi.org/10.3145/epi.2015.nov.06

\section{Introducción}

La interacción con la web semántica (WS) implica pasar de navegar entre documentos a la exploración entre recursos interconectados, de vistas predefinidas como páginas de inicio a vistas complejas generadas dinámicamente como mashups.

Los usuarios finales suelen encontrar difíciles de usar las aplicaciones web semánticas (Heath; Domingue; Shabajee, 2006).

En el contexto de este trabajo, el usuario final se define como un usuario sin o con escasos conocimientos sobre la web semántica. Las tareas de usuario final utilizadas en la Web están ahora claramente definidas y se están convirtiendo en parte de la práctica común de los desarrolladores web. El conocimiento acerca de las tareas en la web semántica es mucho menos claro debido a su novedad (Bakshi; Karger, 2005).

Para explicar cómo hemos abordado este problema, este documento se ha estructurado de la siguiente forma: en la sección 2 se describe una propuesta de tareas de usuario final para la web semántica. La sección 3 presenta un inventario de patrones de interacción de las principales propuestas de conjuntos de patrones publicadas en los últimos años. Este inventario clasifica los patrones de interacción según las tareas de usuario final en las que se pueden utilizar.

En la sección 4 se explican varios ejemplos de cómo los patrones de interacción y el nuevo paradigma de la web semántica pueden mejorar las tareas de usuario final. Y finalmente la sección 5 presenta las conclusiones y el trabajo futuro.

Tareas de usuario final en la WS son: buscar, navegar, anotar, mezclar, mapear, compartir, comunicar y tramitar

\section{Tareas de usuario final en la web semántica}

Se han tomado los trabajos de Heath, Dzbor y Motta (2005), Kellar, Watters y Shepherd (2006), Battle (2006), Mäkelä et al. (2007), Di-Maio (2008) y Sabou et al. (2007), para analizar las propuestas de conjuntos de tareas web y web semántica (WS) para el usuario final (al que consideramos con una visión amplia de usuario online que utiliza todo tipo de servicios y utilidades mediante la web).

Para poder ser estrictamente analizadas consideramos "tareas funcionalmente atómicas" (con funciones únicas e indivisibles) aunque en un contexto real las tareas de usuario de las aplicaciones específicas de la WS se compongan de una mezcla de estas tareas atómicas. El objetivo es definir un conjunto de tareas de usuario que facilite su análisis y que sean combinables para obtener tareas de usuario más complejas y específicas. 


\subsection{Buscar (search)}

Una tarea de búsqueda es aquella que permite a un usuario plantear una consulta y obtener un conjunto de resultados. Incluimos las búsquedas que se pueden diferir o repetir en el futuro, como en los escenarios de supervisión.

En el contexto de la WS, el usuario puede beneficiarse de la semántica implícita cuando se realiza una búsqueda y obtener resultados de mayor precisión y exhaustividad. Por otra parte, el conocimiento capturado en ontologías se puede utilizar con el fin de guiar al usuario a través del proceso de construcción de la consulta, a fin de facilitar la visualización de los resultados en pantalla.

\subsection{Navegar (browse)}

Es la tarea que lleva al usuario a través de una determinada información. En el contexto de los sistemas de información web esto se suele hacer siguiendo los enlaces que conectan la información. Sin embargo, en la WS es posible construir una experiencia de navegación más rica debido a que el modelo subyacente se construye a partir de componentes de menor granularidad, las tripletas formadas por sujeto, predicado y objeto. El conjunto de tripletas constituye un grafo por el que se puede navegar siguiendo los enlaces entre nodos según diferentes criterios.

\subsection{Anotar (annotate)}

Tarea de usuario específica de la WS mediante la cual el usuario describe un recurso, proporcionando propiedades y valores que modelan sus características, sus relaciones con otros recursos, etc. Esta tarea ofrece una nueva descripción o permite complementar una ya existente, modificando o suprimiendo algunos o la totalidad de los atributos.

Un ejemplo de esta tarea es el etiquetado por parte del usuario de un url particular, proporcionando un título o una descripción a un vídeo, etiquetando geográficamente una foto, definiendo un perfil de usuario que incluya detalles y preferencias personales, etc.

La principal particularidad de esta tarea es su notación formal. Como consecuencia, las anotaciones van más allá de las etiquetas informales y ambiguas, convirtiéndose en propiedades y valores que pueden ser especificadas en esquemas y ontologías. Corresponde a la interfaz guiar al usuario por el espacio de conocimiento creado, restringiendo dinámicamente las decisiones que deben tomarse en función de las acciones del usuario anteriores, el contexto de uso y las metas previstas.

\subsection{Mezclar (mashup)}

Reunir y combinar diferentes unidades de información con el fin de conseguir algo más que la simple suma de esas piezas es lo que se entiende por mezclar (o mashup). Ejemplo de una tarea de mashup es mostrar un conjunto de recursos geográficamente situados, como los hoteles cercanos al lugar donde nos encontramos, o recursos con una dimensión temporal como eventos en un calendario que facilitan la gestión de la agenda. En el contexto de la WS esta tarea implica la combinación de dos o más piezas de metadatos sobre recursos comunes con el fin de agregar las descripciones disponibles acerca de ellos.

La principal ventaja de la tecnología semántica para esta tarea es disponer de metadatos semánticos y ontologías que faciliten considerablemente la implementación de algún tipo de ayuda para el usuario durante el proceso de agregación. La asistencia puede variar desde la capacidad de propagar las agregaciones de una propiedad o recurso en particular a todos los usos de esa propiedad en los metadatos mezclados [como la herramienta de mashup Potluck (Huynh; Miller; Karger, 2007)], a la explotación automática de metadatos semánticos utilizando mediciones semánticas y estadísticas [como la herramienta de información de mashup semántico Sig.ma (Cyganiak; Catasta; Tummarello, 2009)].

\subsection{Mapear (map)}

Esta tarea se lleva a cabo cuando el usuario define asignaciones entre términos de diferentes vocabularios. No está restringido a un conjunto particular de recursos, como en el caso de la tarea mashup, ni se usa a nivel de descripciones de recursos. En esta tarea el usuario está trabajando a nivel de vocabularios que pueden ser utilizados en las descripciones de recursos.

Los resultados de una tarea de mapeo pueden utilizarse, por ejemplo, para facilitar o automatizar una mashup, o ambas tareas pueden llevarse a cabo alternativamente y de forma coordinada como un proceso donde el usuario está mezclando -haciendo mashup- un conjunto de descripciones de recursos y durante el proceso se definen asignaciones entre vocabularios.

Indicar que dos etiquetas son equivalentes o relacionar diferentes categorías de productos son ejemplos de este tipo de tarea, que en la WS se pueden incluir cuando el usuario define asignaciones simples entre clases o propiedades definidas en varias ontologías.

\subsection{Compartir (share)}

Esta tarea consiste en subir, publicar, actualizar o eliminar contenido en la web con la intención de ponerlo a disposición de otros usuarios, que pueden acceder al contenido desde el lugar y el momento que cada uno de ellos considere. Esto último es lo que diferencia esta tarea de la de Comunicar que veremos después.

Esta tarea se diferencia de Anotar en el hecho de que lo que se añade, edita o elimina son datos y no metadatos. Estos datos suelen corresponder a diferentes tipos de contenidos que los usuarios quieren compartir, como vídeos, textos o imágenes.

Ejemplos de esta tarea son una publicación en un blog, la compartición de fotos en una red social o dejar un archivo disponible a través de una red. En el contexto de la WS esta tarea, por el hecho de que relaciona datos y no metadatos, es equivalente a la tarea web correspondiente. Sin embargo, puede enriquecerse mediante la indexación de contenido o la generación automática de metadatos que sirvan como entrada para otra tarea. 


\subsection{Comunicar (communicate)}

Consiste en compartir información directamente con otros usuarios sin la intención de ponerla a disposición de terceros. Ejemplos de esta tarea los encontramos en un chat, el envío de un correo-e o una videoconferencia. La comunicación se impulsa desde el emisor, el destinatario sólo tiene que disponer de la herramienta para la comunicación.

\subsection{Tramitar (transact)}

Esta tarea se asocia con las acciones del usuario que provocan un cambio en el estado de una entidad del mundo real o de un recurso en un sistema con el que está interactuando.

Ejemplos de esta tarea son la compra de un libro o la orden de una transferencia de dinero entre cuentas bancarias. La gama de tareas específicas incluidas en esta categoría puede variar mucho en función del sistema interactivo. Cualquier tarea que implica interacción con otros sistemas podría considerarse una transacción.

Aprovechando las tecnologías y metodologías de la web semántica, este tipo de acciones puede mejorar considerablemente la experiencia del usuario (facilitando el rellenado de formularios, adaptando los resultados a las preferencias del usuario y el contexto, etc.).

\subsection{Trabajos relacionados}

Varias definiciones de conjuntos de tareas de usuario final para la Web y para la WS se presentan en los trabajos de Heath, Dzbor y Motta (2005), Kellar, Watters y Shepherd (2006), Battle (2006), Mäkelä et al. (2007), Di-Maio (2008) y Sabou et al. (2007). En un trabajo previo, comparamos estas propuestas de tareas de usuario final con las tareas de usuario final de la WS (Palacios et al., 2014).

\section{Inventario de patrones de interacción}

Un patrón de interacción (Van-Welie, 2007) o patrón de diseño de interfaz de usuario (Toxboe, 2011) es una solución basada en el diseño de un componente software que resuelve un problema de interacción de un usuario final con una interfaz de usuario.

Se ha construido un inventario de patrones de interacción a partir de propuestas de varios autores como las de Tidwell (2011) (94 patrones); Van-Welie (2007) (131 patrones); Toxboe (2011) (54 patrones); Yahoo! (2011) (59 patrones), Crumlish y Malone (2009) (124 patrones); Quince de Infragistics (2012) (50 patrones). Se han estudiado también otros trabajos, entre ellos, los de Van-Duyne, Landay y Hong (2006) o Scott y Neil, 2009).

Un patrón de interacción o patrón de diseño de interfaz de usuario es una solución basada en el diseño de un componente software que resuelve un problema de interacción de un usuario final con una interfaz de usuario

Todos los patrones han sido clasificados siguiendo las tareas de usuario final de la WS comentadas en el apartado anterior y utilizando el modelo conceptual de la figura 1. Muchas de ellas pueden ser mapeadas directamente de la Web tradicional a la WS (por ejemplo, los patrones de Van-Welie para buscar y navegar). Otras veces distintas fuentes explican el mismo patrón dándole diferentes nombres y matices; se han agrupado patrones idénticos o similares en una única propuesta. Los patrones deben ser estudiados en profundidad para adaptarlos a la WS y existe un amplio margen para definir nuevos patrones que implementen la interfaz de las tareas de la WS.

Del análisis realizado concluimos que la contribución de la WS ha de ser más importante para tareas como anotar, mezclar o mapear. Para estas tareas, las más novedosas y

Tabla 1. Propuestas de tareas de usuario final

\begin{tabular}{|c|c|c|c|c|c|c|}
\hline & Heath & Battle & Kellar & Mäkelä & Sabou & Tools \\
\hline Buscar & $\begin{array}{l}\text { Locating, Moni- } \\
\text { toring }\end{array}$ & $\begin{array}{l}\text { Information } \\
\text { seeking }\end{array}$ & Fact finding & $\begin{array}{l}\text { Semantic content } \\
\text { consumption }\end{array}$ & & $\begin{array}{l}\text { Swoogle, Sem- } \\
\text { Search, Falcon-S }\end{array}$ \\
\hline Navegar & Exploring, grazing & $\begin{array}{l}\text { Information } \\
\text { seeking }\end{array}$ & Browsing & $\begin{array}{l}\text { Semantic content } \\
\text { consumption }\end{array}$ & & $\begin{array}{l}\text { Tabulator, exhibit, } \\
\text { mSpace }\end{array}$ \\
\hline Anotar & Asserting & Content update & Maintenance & Content indexing & & Annotea, Faviki \\
\hline Mezclar & $\begin{array}{l}\text { Evaluating, arran- } \\
\text { ging }\end{array}$ & $\begin{array}{l}\text { Information } \\
\text { Synthesis }\end{array}$ & $\begin{array}{l}\text { Information } \\
\text { gathering }\end{array}$ & $\begin{array}{l}\text { Semantic content } \\
\text { consumption }\end{array}$ & & $\begin{array}{l}\text { Sigma, Semantic } \\
\text { Pipes }\end{array}$ \\
\hline Mapear & & Ontology mapping & & & $\begin{array}{l}\text { Folksonomy enrichment, } \\
\text { Word sense desambi- } \\
\text { guation }\end{array}$ & Potluck, Snoggle \\
\hline Compartir & Sharing & $\begin{array}{l}\text { Information } \\
\text { Sharing }\end{array}$ & & & & $\begin{array}{l}\text { Semantic Me- } \\
\text { diaWiki, Twine }\end{array}$ \\
\hline Comunicar & $\begin{array}{l}\text { Notifying, discus- } \\
\text { sing }\end{array}$ & & & & & $\begin{array}{l}\text { Haystack, xOpe- } \\
\text { rator }\end{array}$ \\
\hline Tramitar & Transacting & Action-oriented & & & & $\begin{array}{l}\text { Semantic web } \\
\text { services }\end{array}$ \\
\hline $\begin{array}{l}\text { Not end- } \\
\text { users }\end{array}$ & & & & $\begin{array}{l}\text { Ontology main- } \\
\text { tenance and } \\
\text { publishing }\end{array}$ & Ontology matching & \\
\hline
\end{tabular}


próximas a la WS, existen pocos patrones de interacción actuales que sean aplicables.

En cambio, para tareas como buscar (20 patrones de diferentes conjuntos), navegar (134 patrones), compartir (93 patrones), comunicar (12 patrones) o tramitar (130 patrones) ha sido posible encontrar patrones múltiples existentes que son el resultado de años de trabajo para encontrar soluciones a la interacción con la Web.

Un trabajo futuro será definir nuevos patrones de interacción que sean específicos para la WS y se puedan aplicar en estas nuevas tareas de usuario final. Asimismo, se deben adaptar los existentes a las nuevas características de la WS

Para implementar el inventario de patrones de interacción se ha definido el modelo conceptual de la figura 1.

El objetivo es definir un marco de referencia que permita confluir las propuestas de los conjuntos de patrones estudiados, así como las realizadas en el futuro. Con este inventario se centralizan en un repositorio las propuestas de patrones de interacción para facilitar el trabajo a la comunidad investigadora en temas de $\mathrm{HCl}$ (human computer interaction) y web semántica, y, en general, a toda la comunidad de desarrolladores de interfaces. En el modelo se relacionan los conceptos de tarea de usuario final comentados en el apartado 2 con el de patrón de interacción introducido en el apartado 3, definiendo subclases para la propuesta consolidada de patrones-tipo. En concreto:

- Task: representa las tareas de usuario final de la web semántica;

- InteractionPattern: cada una de las propuestas de patrones de interacción que se han estudiado (a partir de una o de varias propuestas de uno o varios autores). Los patrones son clasificados definiendo subclases para los patrones equivalentes de varias propuestas. Las subclases son instanciadas con cada uno de los patrones;

- SetOfPatterns: cada uno de los conjuntos de patrones propuestos por diferentes autores a la comunidad de desarrolladores de interfaces;

- Author: contiene información de todos los investigadores o empresas que han realizado propuestas en el campo de los patrones de interacción.

Una vez traducido el modelo a RDF, el inventario de patrones se ha hospedado en Rhizomer (Brunetti; García;
Auer, 2013), una plataforma basada en tecnologías de la WS que facilita la publicación de datos semánticos y que permite implementar aplicaciones interactivas propias de la WS.

Así, el inventario de patrones de interacción resultante de este estudio puede visualizarse usando Rhizomer en: http://indagus.udl.cat/iPatternsSemWeb

\section{Mejorar las tareas de usuario final de la web semántica con patrones de interacción}

\subsection{Enriquecer "buscar"}

En la Web los usuarios finales recurren a patrones de interacción como el cuadro de búsqueda Search box o la búsqueda avanzada Advanced search de Van-Welie (figura 2).

Estos patrones de interacción se basan en el uso de formularios de palabras clave con cero o más filtros que ayudan a los buscadores a encontrar estas palabras clave en las páginas web.
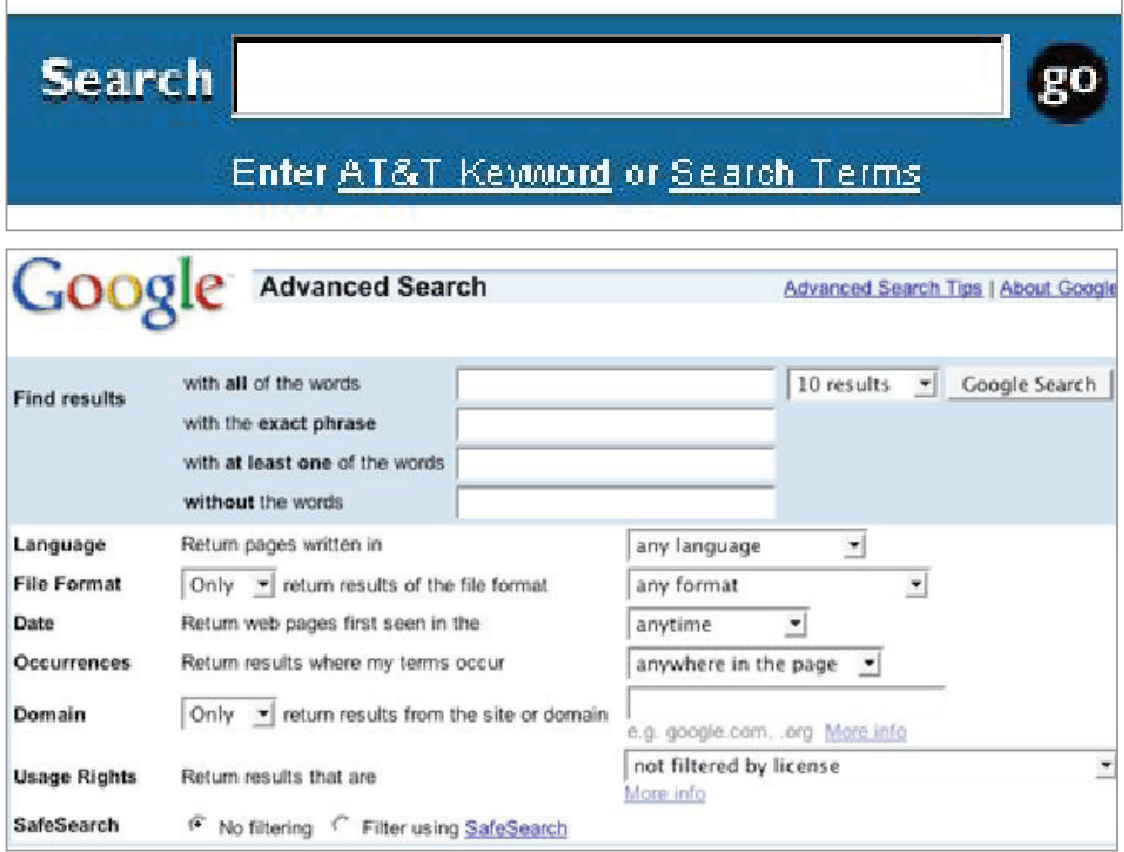

Figura 2. Search y Advanced search de Van-Welie 


\begin{tabular}{|c|c|c|c|}
\hline Select a Topic & & \multirow{2}{*}{\multicolumn{2}{|c|}{ Select a Freebase topic to display here using the suggest box on the left }} \\
\hline La Granja & & & \\
\hline \multicolumn{2}{|l|}{ Select an item from the list: } & \multirow{8}{*}{\multicolumn{2}{|c|}{$\begin{array}{l}\text { La Granja } \\
\text { Architect: Teodoro Ardemans, Filippo } \\
\text { Juvarra, Andrea Procaccini } \\
\text { Opened: } 1741 \\
\text { The Royal Palace of La Granja de San } \\
\text { Ildefonso (Spanish: Palacio Real de La } \\
\text { Granja de San Ildefonso) is an } 18 \text { th } \\
\text { century palace in the small town of San } \\
\text { Ildefonso in the hills near Segovia, } \\
80 \text { km north of Madrid, central Spain, } \\
\text { formerly the summer res... }\end{array}$}} \\
\hline La Granja & Building & & \\
\hline La Granja & Musical Artist & & \\
\hline La Granja & City/Town/Nillage & & \\
\hline La Granja & TV Program & & \\
\hline La Granja humana & Musical Release & & \\
\hline La Granja humana & Musical Album & & \\
\hline CD La Granja & Football team & & \\
\hline Estadio La Granja & Structure & \multirow{4}{*}{ Building, Landscape project, Structure } & $\approx \oplus 0$ \\
\hline La Granja de la Costera & City/Town/Nillage & & \\
\hline La Granja d'Escarp & Topic & & \\
\hline view more & & & \\
\hline
\end{tabular}

Figura 3. Search en freebase.com
Otra alternativa es proporcionar una Faceted view si los metadatos por los que se está navegando son homogéneos. Además de la estructura de metadatos explícita, también es posible aprovechar las ontologías subyacentes a fin de obtener nuevos vínculos entre los recursos utilizando mecanismos de inferencia, agrupación o consultas semánticas a otras fuentes. $Y$ todo esto dinámicamente, proponiendo productos relacionados con los que antes hemos visitado.

Un ejemplo de esto son los patrones de interacción Faceted

En la WS, el conocimiento capturado en ontologías puede guiar al usuario a través del proceso de construcción de consultas, lo que facilita la consulta by example o los resultados de visualización. Además, el contenido semántico puede ayudar a generar dinámicamente el formulario que ayude en la búsqueda.

En el ejemplo de freebase.com (figura 3), después de que el usuario introduzca el tema, se muestra una lista de posibilidades con una explicación de los significados del tema.

\subsection{Enriquecer "navegar"}

En la web, el usuario se mueve por la información que se muestra a través de enlaces como en el patrón de interacción Main navigation de Van-Welie, donde un menú siempre visible se coloca en una posición fija de la página (figura 4).

Explicamos en la sección 2.2 que, en el contexto de la WS, es posible construir una experiencia de navegación más rica gracias al modelo subyacente que, construido como una combinación de tripletas, forman un grafo por el que se puede navegar siguiendo los enlaces entre sus nodos. navigation o Faceted view de Van-Welie (figura 5).

\subsection{Enriquecer "anotar"}

Anotar es una tarea que permite al usuario final asociar información -anotar- en forma de etiquetas, atributos, propiedades o relaciones.

Es una de las tareas más nuevas y no existen casi patrones de interacción para implementarla. Un ejemplo puede ser gestionar la propiedad intelectual usando los patrones Copyleft, Copyright o Creative commons de Crumlish y Malone (2009).

Un ejemplo de uso en el contexto de la web semántica es la Semantic forms extension de la Semantic MediaWiki (Krötzsch; Vrandečić; Völkel, 2006), que aprovecha los modelos semánticos subyacentes para estructurar tipos disponibles, propiedades y sus valores.

Pero una de las áreas de aplicación más interesantes del contenido semántico son los snippets: unas pocas líneas de texto que aparecen debajo de cada resultado de búsqueda y que tienen el propósito de ayudar al usuario a decidir qué enlace escoger.

Los principales buscadores recomiendan el uso de snippets enriquecidos, información detallada destinada a ayudar a los usuarios con consultas específicas. Con ellos, los motores de búsqueda pretenden entender mejor el contenido de las páginas web.

El enriquecimiento semántico de 'rich snippets' puede ser implementado utilizando microformatos 0 , más recientemente $y$ especialmente en html5, con microdatos o RDFa, la versión de RDF que se incrusta dentro de html5.

En este ámbito se debe mencionar la experiencia de Schema.org ${ }^{1}$, la propuesta de los principales motores de búsqueda (Bing, Google, Yahoo!, Yandex) para construir un único marco conceptual que permita el enriquecimiento semántico de sitios web o la interoperabilidad de bases de datos (figura 6). 


\subsection{Enriquecer "mezclar"}

Un sitio web generado como el resultado de un mashup (mezcla) es la combinación de contenido proveniente de varias fuentes independientes.

Mashup es una tarea más reciente que las tradicionales navegar y buscar; por ello encontramos muy pocos patrones de interacción para implementarla. Uno de los pocos ejemplos es el patrón Geo-mashing de Crumlish y Malone (2009).

El contenido semántico -datos estructurados, ontologías, herramientas de razonamiento- ayuda a automatizar el proceso de mezcla, pudiendo generar automáticamente páginas mashup para un sitio web.

En el ejemplo que se muestra en la figura 7, el contenido propio del sitio web de la $B B C$ se enriquece con contenidos externos de Wikipedia y MusicBrainz. Los periodistas de la $B B C$ no tienen la responsabilidad de mantener el contenido de la Wikipedia o MusicBrainz a pesar de que son contenidos mostrados en su web. Sin embargo, esta forma de trabajar tiene un buen efecto secundario: los periodistas de la $B B C$ están involucrados en el proyecto colaborativo de la Wikipedia para mantener el contenido al día.

\subsection{Enriquecer "mapear"}

La tarea de mapear es rara en el contexto de la Web. Un ejemplo es la definición de asignaciones entre hashtags en tagdef.com.

Las herramientas de la WS pueden relacionar etiquetas, términos, conceptos o clases. Un mapeo enriquecido semánticamente puede facilitar o automatizar los mashups.

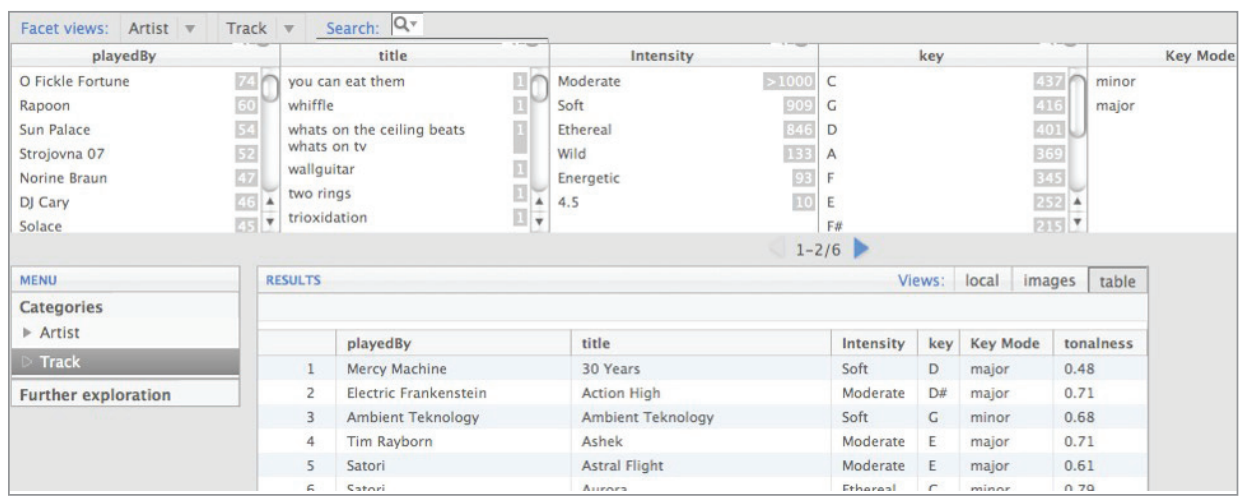

Figura 5. Ejemplo de Faceted navigation o Faceted view de Van-Welie

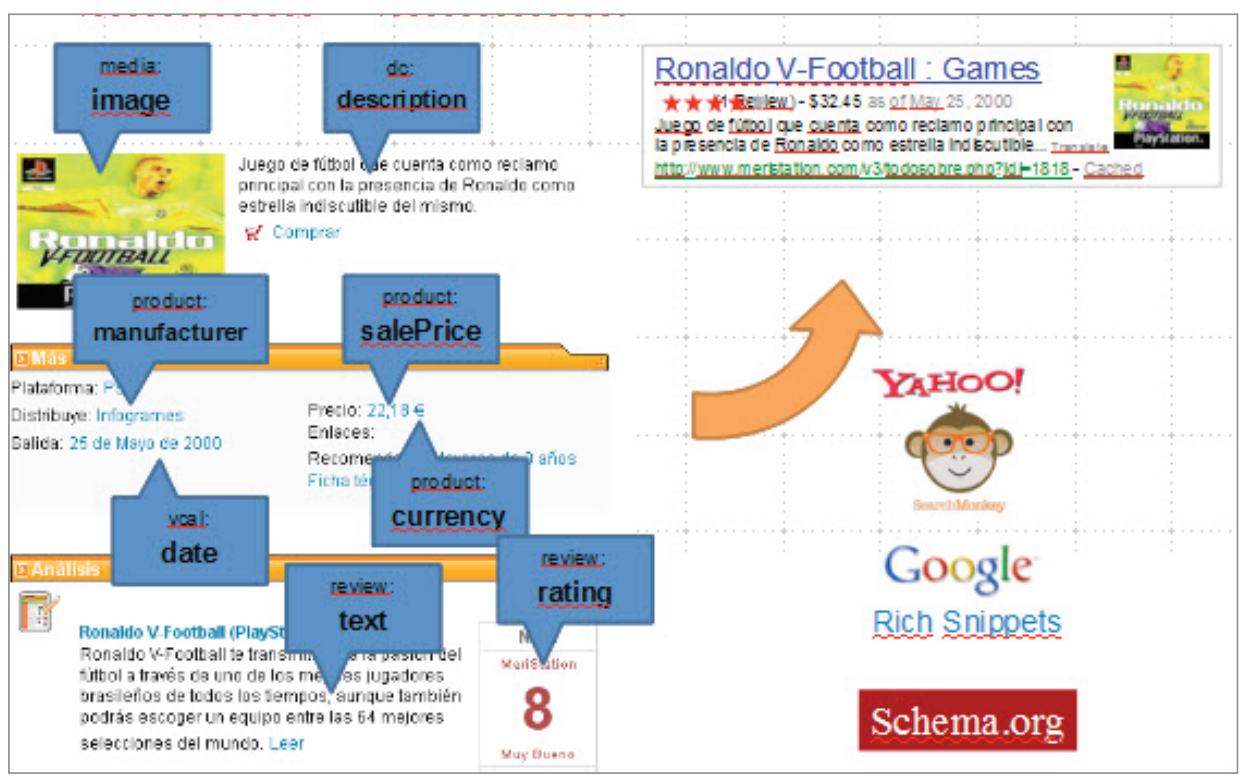

Figura 6. Ejemplo de snippets

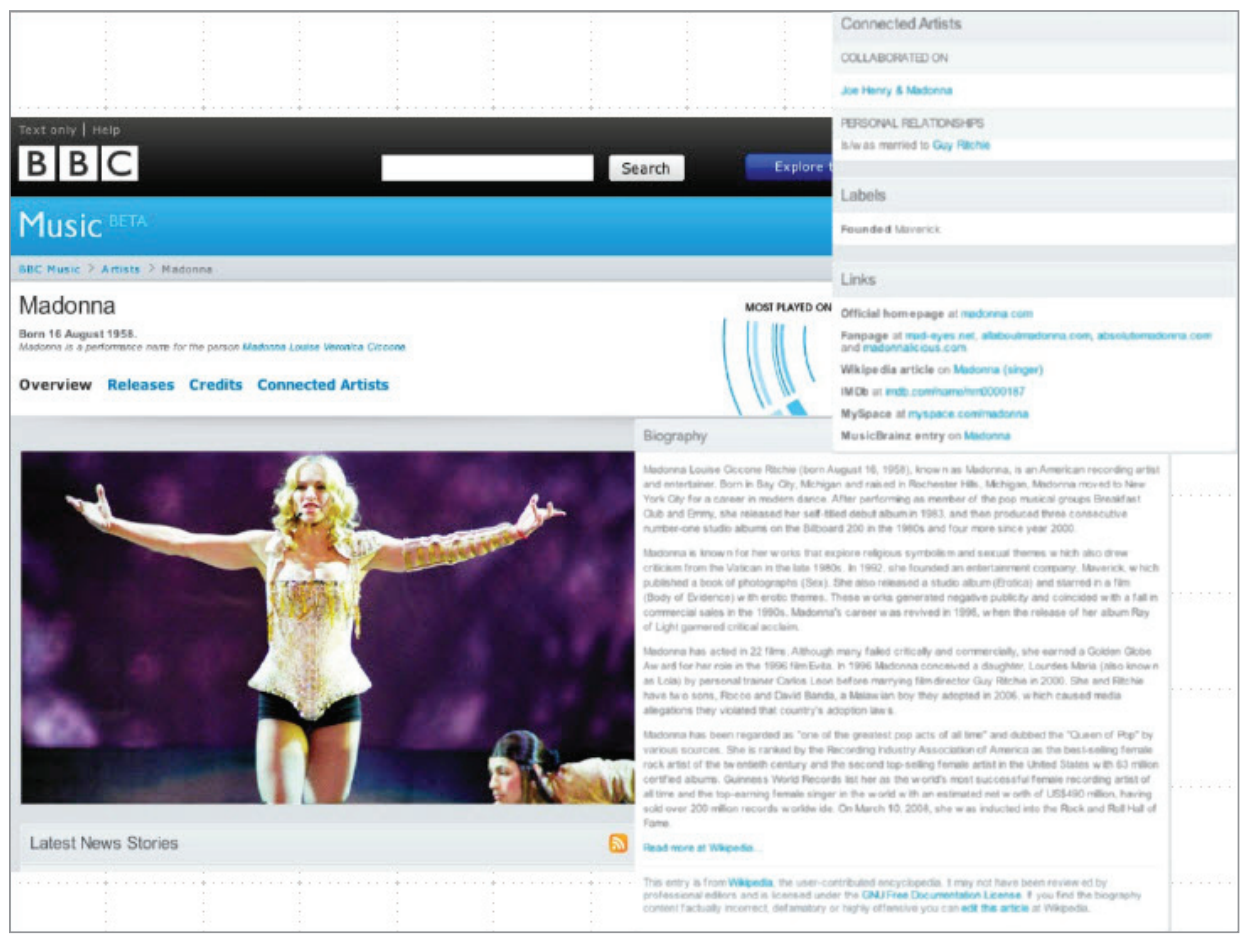

Figura 7. Ejemplo de mashup de la $B B C$ 


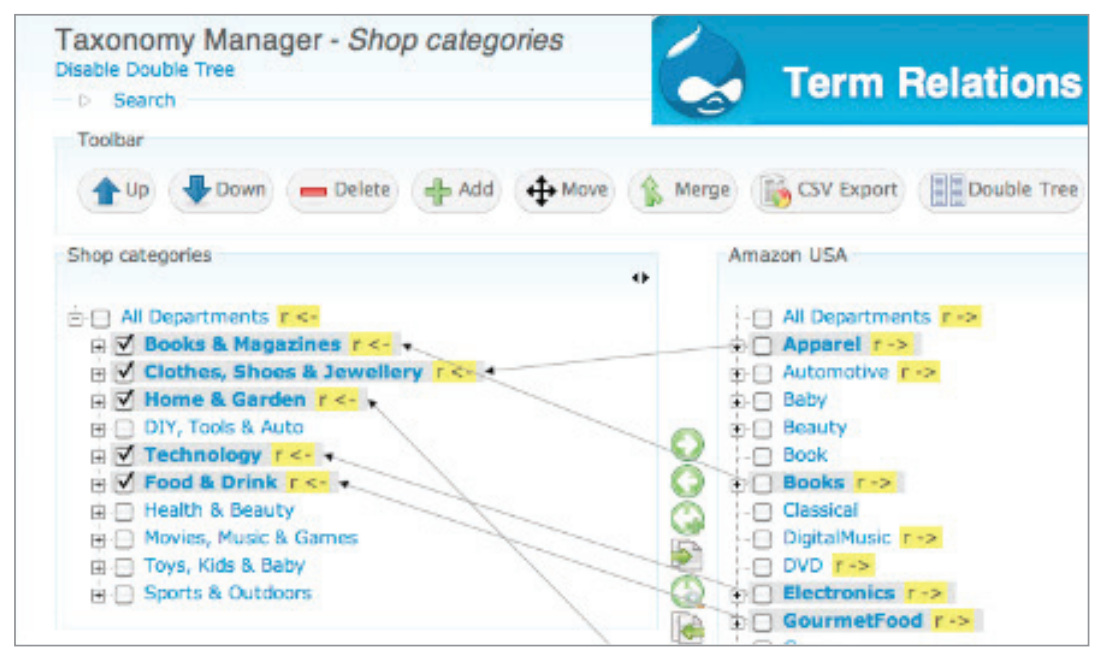

Figura 8. Ejemplo de mapear en Drupal

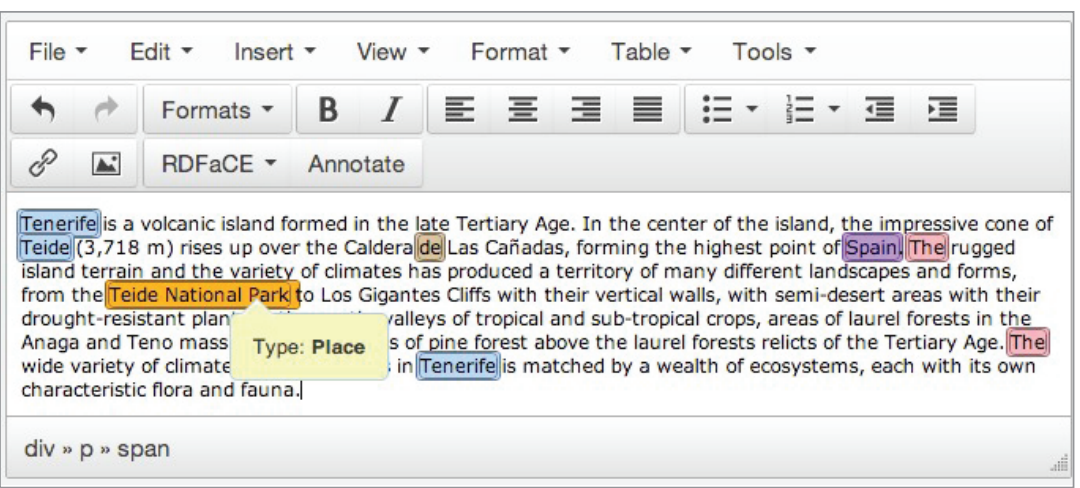

Figura 9. Herramienta RDFaCE

http://rdface.aksw.org

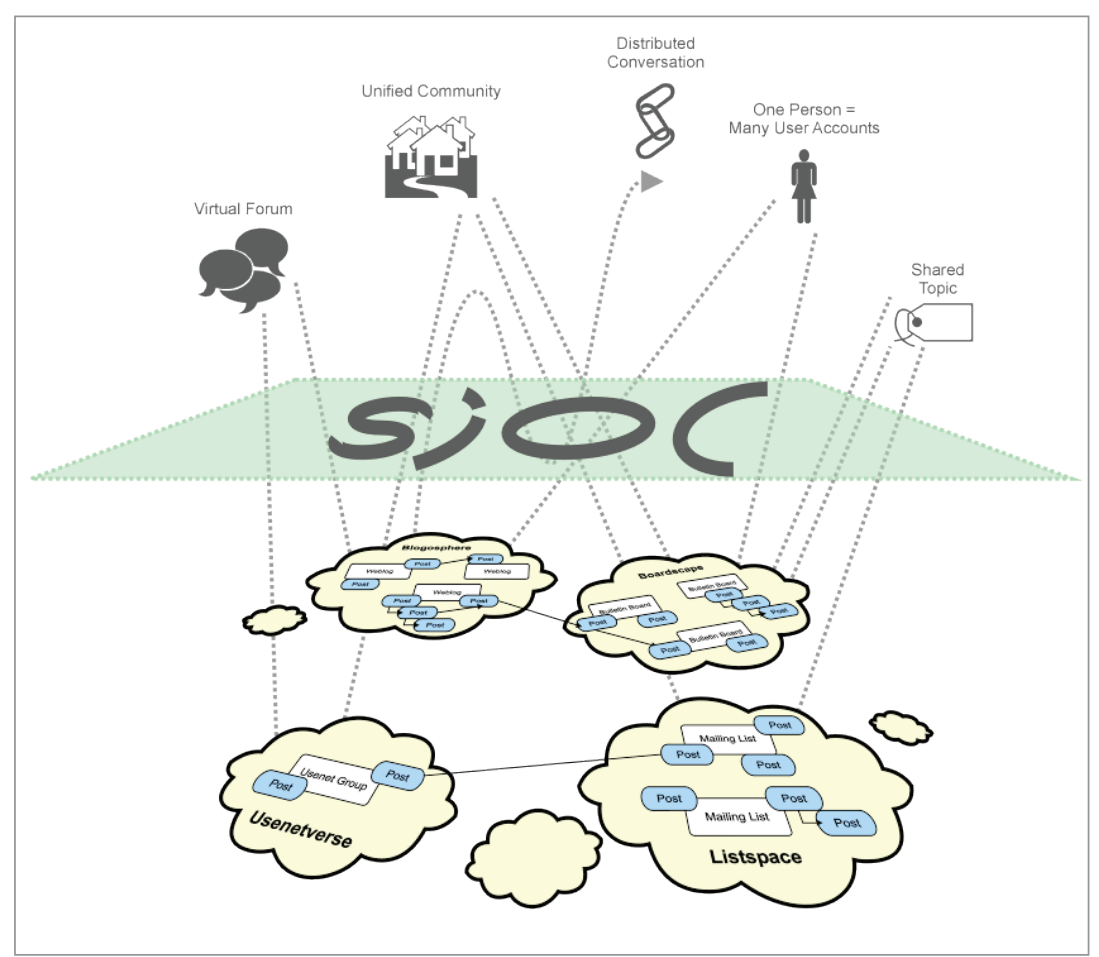

Figura 10. Enlazado semántico de comunidades online mediante SIOC
En el ejemplo de la figura 8, un usuario de Drupal puede asignar las categorías de productos de su tienda online con las categorías de una empresa de comercio electrónico como Amazon usando el administrador de taxonomías de Drupal.

\subsection{Enriquecer "compartir"}

Existe multitud de patrones de interacción para la tarea compartir. En la WS estos patrones se enriquecen mediante la indexación del contenido compartido para su anotación semántica o mediante herramientas interactivas. Por ejemplo, la herramienta RDFaCE (figura 9) extiende el editor de html TinyMCE basado en el principio wysiwyg (what you see is what you get) para que el usuario pueda, además de definir el contenido a nivel visual (lo que se ve), definir su semántica (lo que significa). Para ello propone el principio wysiwym (what you see is what you mean). Esto supone mejoras por ejemplo en el posicionamiento en buscadores.

\subsection{Enriquecer "comunicar"}

En el caso de la tarea comunicar, los patrones de interacción ya establecidos se pueden mejorar enriqueciendo los mensajes intercambiados con anotaciones semánticas generadas de forma automática. De esta forma se pueden ofrecer nuevas formas de ver una comunicación, por ejemplo un intercambio de tweets, en base a los conceptos mencionados y sus interrelaciones a nivel semántico.

Es posible brindar al usuario vistas integradas de conversaciones a través de diferentes servicios de comunicación (listas de correo, blogs, servicios de chat...) mediante una capa común como SIOC, una ontología para el enlazado semántico de comunidades online (figura 10). http://sioc-project.org

\subsection{Enriquecer "tramitar"}

Finalmente, también es posible enriquecer la experiencia de usuario en el caso de tramitar. Por ejemplo, utilizando el esquema de datos universal Schema. org, promocionado por los principales buscadores, como se dijo, ya es posible anotar correos electrónicos y que clientes de correo como GMail utilicen esas anotaciones semánticas para facilitar al usuario ciertos trámites. Por ejemplo, 
anotar que el correo es sobre un vuelo y especificar detalles como aerolínea, aeropuerto de salida, horario..., y enriquecer la visualización del correo con enlaces para el registro del vuelo (figura 11).

\section{Conclusiones y trabajo futuro}

Para hacer llegar la web semántica (WS) a los usuarios finales, es necesario empezar por hacer las aplicaciones de

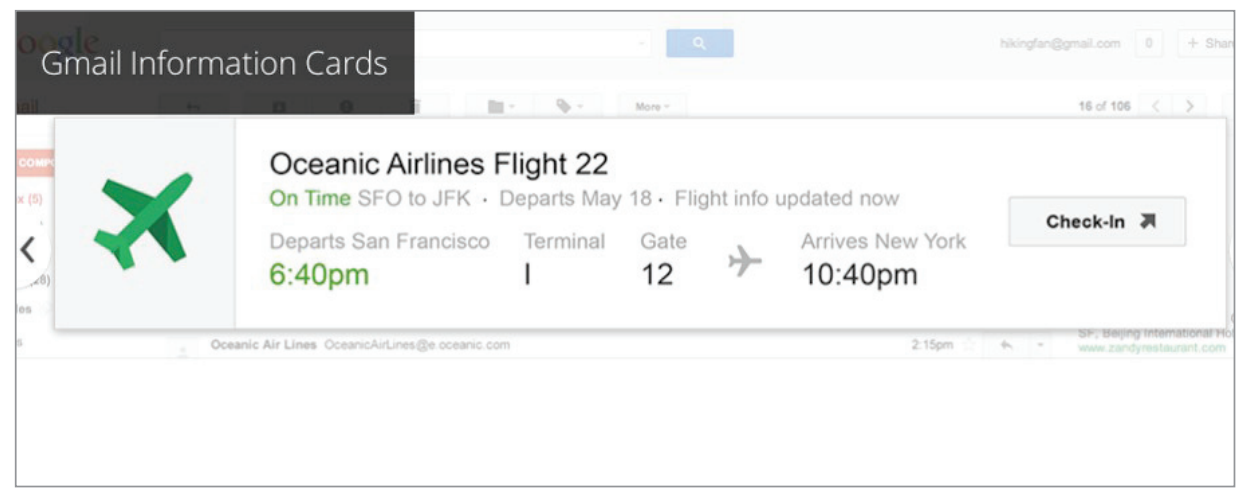

Figura 11. Ejemplo de enriquecimiento en Gmail

WS más usables. En este sentido, es práctica común en la disciplina de la interacción persona-ordenador empezar por definir un conjunto de tareas de usuario habituales en un contexto determinado, por ejemplo los sistemas de información web. Esto permite establecer directrices y patrones de interacción para ese dominio.

Sin embargo, antes es preciso especificar el perfil del usuario y el contexto de uso. En este caso, como el objetivo es contribuir a la adopción generalizada de la WS, el usuario destino es un "usuario final sin conocimientos específicos de WS". Y el contexto es el de las aplicaciones web, eso sí, basadas en tecnologías de WS.

Con el usuario y contexto definidos, se han determinado entonces las tareas del usuario. Para tener en cuenta una amplia gama de ellas, no se ha restringido el análisis inicial a las ya presentes en la WS actual. Además de estudios existentes de tareas de usuario de WS, se han considerado tareas de inventarios existentes para sistemas de información online.

Como resultado de este análisis se ha propuesto un conjunto de tareas que incluye: navegar, anotar, mezclar, mapear, compartir, comunicar y tramitar. Cada una de estas tareas se ha presentado desde una perspectiva general, y luego teniendo en cuenta las oportunidades que las tecnologías de WS ofrecen para mejorar la experiencia de usuario.

En base a este conjunto de tareas de usuario en la WS, también se ha creado un inventario de patrones de interacción apropiados para cada una de ellas. Se ha partido de los repositorios de patrones de interacción más populares, que se han analizado bajo la perspectiva de la WS y clasificado según las tareas de usuario propuestas para ella.

Se ha construido un modelo para los patrones de interacción que nos ayude a automatizar el proceso de estudio, a centralizar las propuestas de los conjuntos de patrones en un único repositorio y a servir de base para propuestas de autogeneración de interfaces de usuario para la WS.

El modelo se ha traducido a RDF y se han centralizado todas las propuestas de patrones en Rhizomer, una plataforma web que gestiona la información con tecnologías de la WS.

Lo siguientes pasos se centran ahora en modelar los principales patrones de interacción del inventario utilizando ontologías que permitan representar las características de cada uno utilizando los mismos principios básicos. El modelo nos debe permitir validar la clasificación de los patrones y las

decisiones sobre equivalencias entre patrones. También se deben modelar las necesidades de interacción para poder enlazar necesidades con patrones. De esta forma pensamos que se podrá desarrollar un recomendador que asista durante la creación de interfaces de usuario, o incluso automatizar la generación de la interfaz a partir de los datos semánticos y las tareas de usuario a las que se quiere dar soporte a través de la interfaz.

\section{Notas}

\section{Disponible en:}

http://schema.org

y con el soporte de los buscadores Bing, Google, Yahoo! y Yandex.

\section{Bibliografía}

Bakshi Karun; Karger, David (2005). "End-user application development for the semantic web". En: Semantic desktop

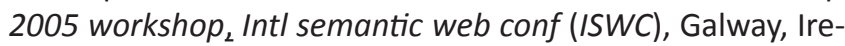
land.

http://ceur-ws.org/Vol-175/36_bakshi_endusersemdesk_ final.pdf

Battle, Lisa (2006). "Preliminary analysis of users and tasks for the semantic web". En: Semantic web user interaction workshop, Intl semantic web conf (ISWC), Athens, GA, USA. http://www.designforsearch.com/files/lb_users_tasks_ semantic_web.pdf

Brunetti, Josep-Maria; García, Roberto; Auer, Sören (2013). "From overview to facets and pivoting for interactive exploration of semantic web data". International journal of semantic web and information systems, v. 9, n. 1, pp. 1-20. http://rhizomik.net/html/ roberto/papers/IJSWISSIVisualisationandInteraction.pdf http://dx.doi.org/10.4018/jswis.2013010101

Crumlish, Christian; Malone, Erin (2009). Designing social interfaces. O'Reilly; Yahoo Press. ISBN: 9781491919859

Cyganiak, Richard; Catasta, Michele; Tummarello, Giovanni (2009). "Towards Ecsse: live web of data search and integration". En: Semantic search 2009 workshop, Madrid, Spain. http://richard.cyganiak.de/2008/papers/ecsse-semsearch2009. $p d f$

Di-Maio, Paola (2008). "Toward global user models for semantic technologies: emergent perspectives". En: Procs of 
the $3^{\text {rd }}$ Asian semantic web conf., ASWC'08, Bangkok, Thailand, pp. 141-152.

http://lists.w3.org/Archives/Public/public-semwebui/2009Jan/att-0006/global_user_models_ethnography.pdf

Heath, Thomas; Domingue, John; Shabajee, Paul (2006). "User interaction and uptake challenges to successfully deploying semantic web technologies". En: Procs of the $3^{\text {rd }}$ Intl semantic web user interaction workshop, Athens, Georgia, USA. http://tomheath.com/papers/heath-swui2006-userinteraction-uptake-challenges.pdf

Heath, Thomas; Dzbor, Martin; Motta, Enrico (2005). "Supporting user tasks and context: Challenges for semantic web research". En: Procs of ESWC2005 Workshop on end-user aspects of the semantic web (UserSWeb).

http://kmi.open.ac.uk/events/usersweb/papers/11_heath final.pdf

Huynh, David; Miller, Robert; Karger, David (2007). "Potluck: Data mash-up tool for casual users". En: $6^{\text {th }}$ Int/ semantic web conf (ISWC), Springer. Lecture notes in computer science, v. 4825, pp. 239-252.

http://dx.doi.org/10.1007/978-3-540-76298-0_18

Infragistics (2012). Quince.

http://quince.infragistics.com/html/AllPatterns.aspx

Kellar, Melanie; Watters, Carolyn; Shepherd, Michael (2006). "A goal-based classification of web information tasks". En: Procs of the $69^{\text {th }}$ Annual meeting of the American Society for Information Science and Technology, v. 43, n. 1, pp. 1-22. http://eprints.rclis.org/8781

http://dx.doi.org/10.1002/meet.14504301121

Krötzsch, Markus; Vrandečić, Denny; Völkel, Max (2006). "Semantic media wiki". En: The semantic web - ISWC 2006, Heidelberg, Germany. Lecture notes in computer science, v. 4273, pp. 935-942.

http://dx.doi.org/10.1007/11926078_68

Mäkelä, Eetu; Viljanen, Kim; Alm, Olli; Tuominen, Jouni;
Valkeapää, Onni; Kauppinen, Tomi; Kurki, Jussi; Sinkkilä, Reettaa; Känsälä, Teppo; Lindroos, Robin; Suominen, Osma; Ruotsalo, Tuukka; Hyvönen, Eero (2007). "Enabling the semantic web with ready-to-use web widgets". En: FIRST - First industrial results of semantic technologies workshop, at the ISWC+ASWC Conf., Busan, Korea.

http://ftp.informatik.rwth-aachen.de/Publications/CEURWS/Vol-293/paper5.pdf

Palacios, Alfons; García, Roberto; Oliva, Marta; Granollers, Toni (2014). "Semantic web end-user tasks". En: Interaccion 2014. Procs of the XV Intl conf on human computer interaction.

http://dx.doi.org/10.1145/2662253.2662299

Sabou, Marta; García, Jorge; Angeletou, Sofia; D’Aquin, Mathieu; Motta, Enrico (2007). "Evaluating the semantic web: A task-based approach". En: The $6^{\text {th }}$ Int/ semantic web conf and The $2^{\text {nd }}$ Asian semantic web conf. Lecture notes in computer science, v. 4825, pp.423-437.

http://dx.doi.org/10.1007/978-3-540-76298-0_31

Scott, Bill; Neil, Theresa (2009). Designing web interfaces. O'Reilly Media. ISBN: 9780596516253

Tidwell, Jenifer (2011). Designing interfaces, $2^{\text {nd }}$ ed. O'Reilly Media. ISBN: 9781449379704

Toxboe, Anders (2011). "User interface desing pattern". UI patterns.

http://ui-patterns.com

Van-Duyne, Douglas; Landay, James; Hong, Jason (2006). The design of sites: Patterns for creating winning websites. Prentice Hall. ISBN: 9780131345553

Van-Welie, Martijn (2007). "A pattern library for interaction design". Welie.com.

http://www.welie.com

Yahoo! (2012) "The Yahoo! Design pattern library". Yahoo! Developer network.

http://developer.yahoo.com/ypatterns

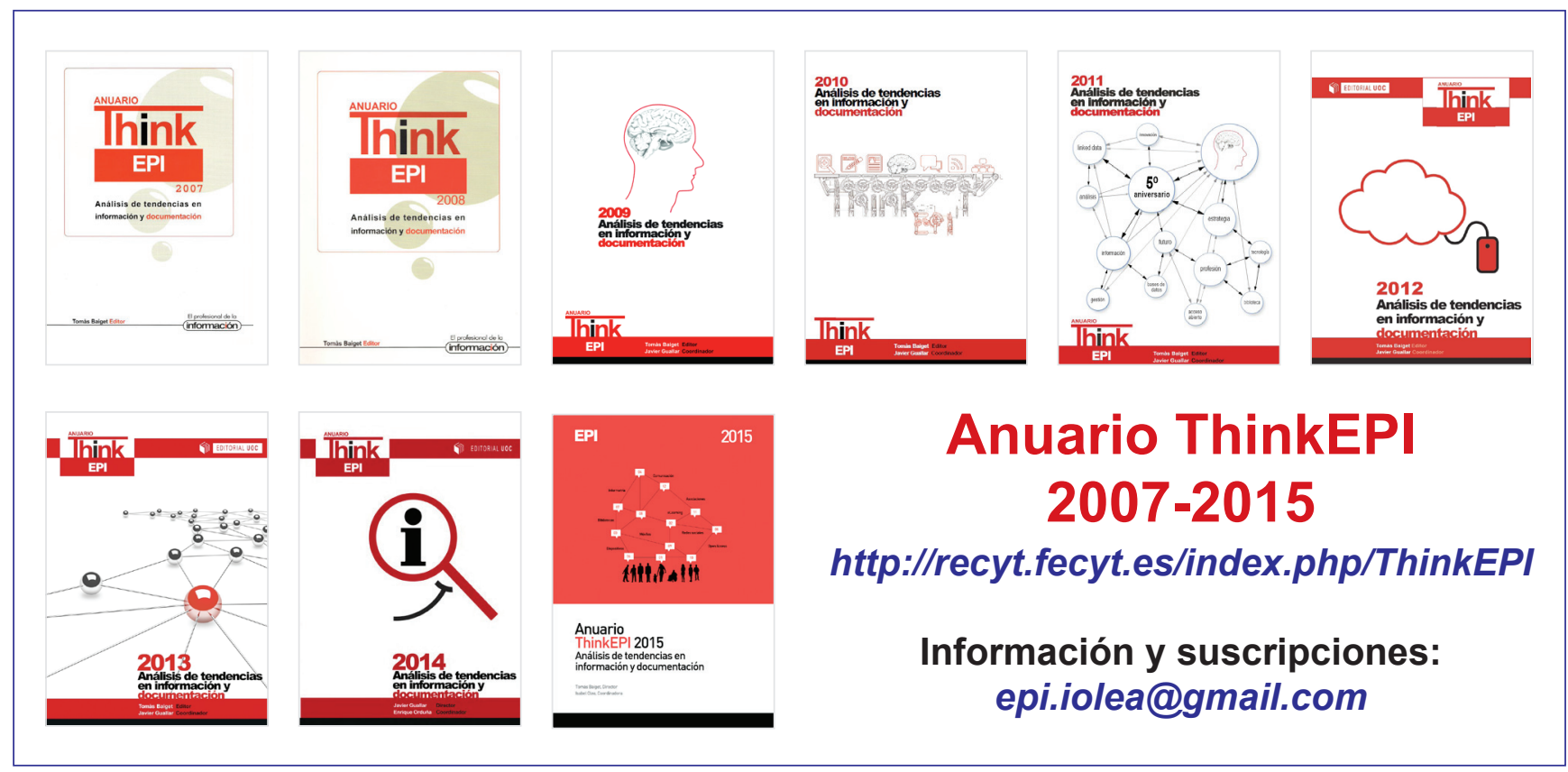

